\title{
A Study of the Mechanical Properties of Au Nanomeshes
}

\author{
Dongjing Li, ${ }^{1}$ Ganming Li, ${ }^{1}$ Qing Wan, ${ }^{1}$ Wuxiong $\mathrm{Xu},{ }^{1}$ Jianghua Liu, ${ }^{1}$ and Zeping $\mathrm{Li} \mathbb{D}^{1,2}$ \\ ${ }^{1}$ School of Electronic Information and Engineering, Hubei University of Science and Technology, Xianning 437005, China \\ ${ }^{2}$ School of Optical and Electronic Information, Huazhong University of Science and Technology, Wuhan 430074, China
}

Correspondence should be addressed to Zeping Li; d201477516@alumni.hust.edu.cn

Received 24 February 2020; Revised 14 April 2020; Accepted 27 April 2020; Published 25 May 2020

Academic Editor: Zhi Li Xiao

Copyright (c) 2020 Dongjing Li et al. This is an open access article distributed under the Creative Commons Attribution License, which permits unrestricted use, distribution, and reproduction in any medium, provided the original work is properly cited.

\begin{abstract}
We study the mechanical behavior of Au nanomeshes (AuNMs) based on finite element analysis (FEA) simulation and deformation tests. The simulated results of mechanical flexibility indicate that polyethylene terephthalate (PET) substrate can release the stress of AuNMs under mechanical stretching and bending, the displacement of stretched AuNMs yields a $2 \%$ promotion, and the displacement of bent AuNMs yields a 3.5\% promotion under buffering of PET substrate at $5 \mathrm{GPa}$ yield strength of nanoscale $\mathrm{Au}$. The stress and displacement distribution of the AuNMs/PET is demonstrated and analyzed. The further deformation tests of AuNMs under compressive and tensile loading indicate that the simulation data are in good agreement with experimental results. This paper is conducive to understanding the mechanical behavior and corresponding structural response and structural fracture dynamics of AuNMs.
\end{abstract}

\section{Introduction}

Traditional electrode materials, such as indium tin oxide (ITO), are easy to crack in the process of mechanical deformation due to the brittleness of its ceramics, which leads to the degradation of conductivity and device performance [1-4]. Metallic nanomeshes have attracted much interest in flexible optoelectronic devices due to their properties of simultaneous good optical transmittance, electrical conductivity, and mechanical flexibility [5-8]. However, the mechanical flexibility of metallic nanomeshes is of interest for theoretical and technical considerations; morphological change of metallic nanomeshes is likely to lead to the unreliable performance of devices under different levels of deformation, which has been proved to be challenging [9-11]. Due to good biocompatibility and long-term electrical stability without corroding through oxidation or sulfidation reactions of metallic gold $(\mathrm{Au})[12,13]$, we chose $\mathrm{Au}$ for the study here.
In the present paper, the mechanical simulation of AuNMs was carried out using FEA and considering the performance of electrical conductivity under mechanical deformation. The stress distribution of AuNMs with and without PET film during mechanical stretching and bending was investigated, and the stress and displacement distribution of the AuNMs/PET is demonstrated. Then, the deformation tests of AuNMs under compressive and tensile loading were analyzed by comparing the simulated data with experimental results. Finally, the cyclic compressive and tensile bending tests revealed good mechanical stability of AuNMs.

\section{Methods}

2.1. Computational. We investigated the mechanical and structural response of AuNMs under mechanical deformation based on FEA simulation [14-16]. In the following simulation, we set the yield strength of nanoscale gold as $5 \mathrm{GPa}$ and set the thickness of AuNMs and PET to constant values 


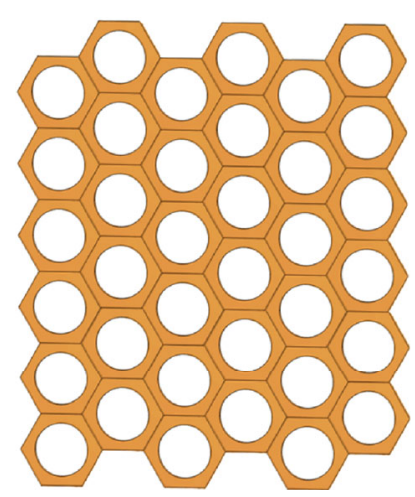

(a)

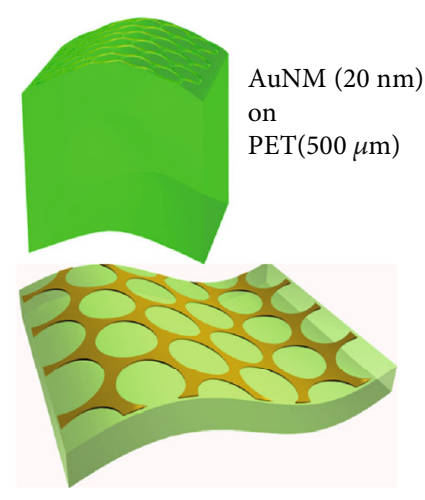

(b)

Figure 1: The schematic drawing in simulation (a) AuNMs and (b) AuNMs/PET.

of $20 \mathrm{~nm}$ and $500 \mu \mathrm{m}$, respectively, which is consistent with that of the sample in the test. Further, parameters of material properties were applied from published experimental data $[17,18]$. The numerical models of AuNMs and AuNMs/PET analyzed in the following simulation are shown in Figures 1(a) and 1(b), respectively.

2.2. Experimental. As a comparison, the AuNMs were fabricated by the well-known NSL technique described in our previous work [19]. In brief, a self-assembled monolayer of polystyrene spheres (PS, Aladdin Co., Ltd.) with a diameter of $1 \mu \mathrm{m}$ was deposited onto a PET film with a thickness of $500 \mu \mathrm{m}$ on the glass substrate, which was cleaned with isopropanol and deionized water. Then, a hexagonally close-packed monolayer of PS was formed as the following template $[20,21]$. The reactive ion etching (RIE, etching gases: $\mathrm{O}_{2}$ and $\mathrm{CHF}_{3}$ ) method was used to form gaps between PS by reducing the diameter of PS, followed by the deposition of metallic nanomeshes of $2 \mathrm{~nm}$ Ti buffer layer and $20 \mathrm{~nm} \mathrm{Au}$ in the gaps between the PS using electron-beam evaporation. Finally, the metallic nanomeshes on the substrate were yielded after PS were removed by sonication.

The self-assembled monolayer of PS with a diameter of $1 \mu \mathrm{m}$ is shown in Figure 2(a), and the typical AuNMs with $\sim 160 \mathrm{~nm}$ average interaperture wire width and $\sim 20 \mathrm{~nm}$ thickness (Figure 2(b)) were adopted in the following tests. The AuNMs prepared show a uniform periodic nanostructure consistent with that of the AuNM model in the simulation.

\section{Results and Discussion}

The application of the metallic nanomeshes always has been looking forward to excellent flexibility. To reveal the flexibility inside, the mechanical flexibility of AuNMs was investigated by examining the change in Von Mises stress distribution of AuNMs under stretching. Here, we compared the flexibility properties of AuNMs with or without PET substrate, which are shown in Figures 3 and 4.

When AuNMs (Figure 3(a)) and AuNMs/PET (Figure 3(b)) are stretched with one side fixed and the other side stretched in $-y$ direction, both of the maximum and minimum stress values appear in the middle of the side length of the hexagonal cell, which are also the maximum and minimum points of force. The results are the same as in Figure 4. When AuNMs (Figure 3(a)) and AuNMs/PET (Figure 3(b)) are applied maximum stress of $5 \mathrm{GPa}$, the stretched displacement of AuNMs increases from $1.356 \times$ $10^{-7} \mathrm{~m}$ without PET in Figure $3(\mathrm{a})$ to $1.3831 \times 10^{-7} \mathrm{~m}$ in Figure 3(b). So, the stretched displacement of AuNMs yields a $2 \%((1.3831-1.356) / 1.356)$ promotion under the buffering of PET substrate.

Likewise, when AuNMs (Figure 4(a)) and AuNMs/PET (Figure 4(b)) are applied maximum stress of $5 \mathrm{GPa}$ and bent with one side fixed and the other side bent in $-z$ direction, the bent displacement of AuNMs increases from $2.6302 \times 10^{-5} \mathrm{~m}$ without PET in Figure 4(a) to $2.721 \times 10^{-5} \mathrm{~m}$ in Figure 4(b). The displacement of bent AuNMs yields a 3.5\% ((2.7212.6302)/2.6302) promotion under the buffering of PET substrate. Notice that the bendability of AuNMs in the vertical direction is larger than the stretchability of AuNMs in the horizontal direction.

The AuNMs supported by the PET substrate show improved tensile strain tolerance, which results in the fact that PET releases the stress in AuNMs under mechanical stretching and bending. It is worth noting that AuNMs with the hexagonal unit cell permits large reversible deformation under strains applied in certain axes due to the axisymmetric circular hole. The supported nature of the PET substrate greatly improves PET substrate stability of AuNMs under bending.

The AuNMs usually need to be placed on a flexible irregular surface for imaging electrical signal. Herein, the flexible PET (thickness $\sim 500 \mu \mathrm{m}$ ) film is used to represent flexible irregular surfaces in the following simulation. The stress and displacement distribution of the AuNMs/PET is demonstrated and analyzed in Figure 5. Different from the previous stretching method, both sides of PET in this model are stretched in $y$ and $-y$ directions, respectively, by equal $4 \times$ $10^{5} \mathrm{~N} / \mathrm{m}^{2}$ force, which results in the deformation of PET and AuNMs. The stress and displacement distribution of the AuNMs/PET is demonstrated. 


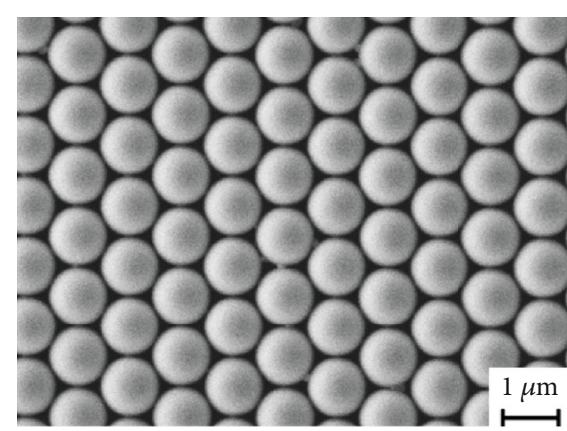

(a)



(b)

FIgURE 2: Preparation of AuNMs: (a) SEM image of the monolayer PS ( $1 \mu \mathrm{m}$ diameter); (b) SEM image of AuNMs ( 160 nm average interaperture wire width).

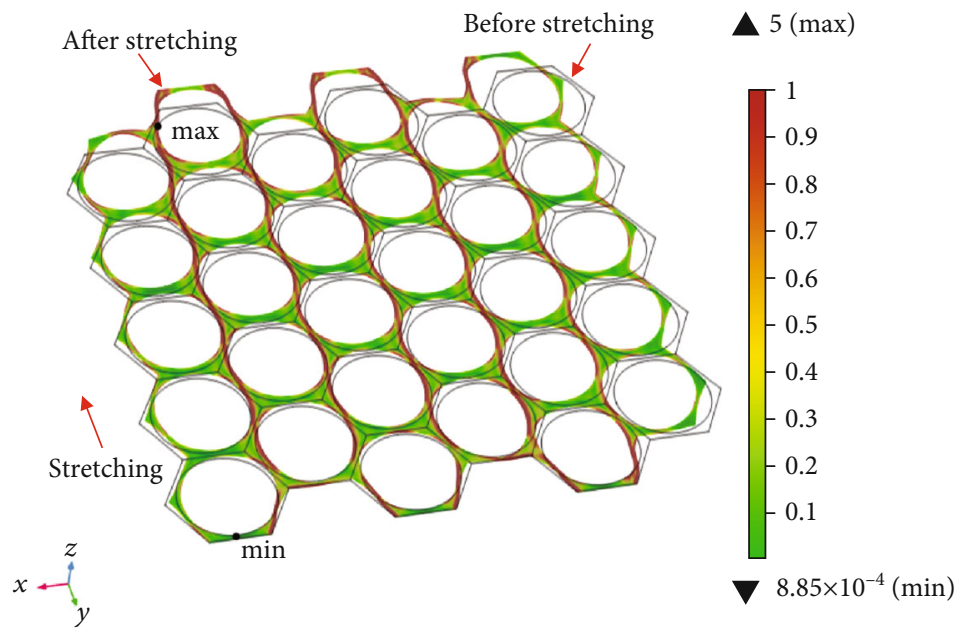

(a) Von Mises stress (GPa) at displacement $=1.356 E-7 \mathrm{~m}$

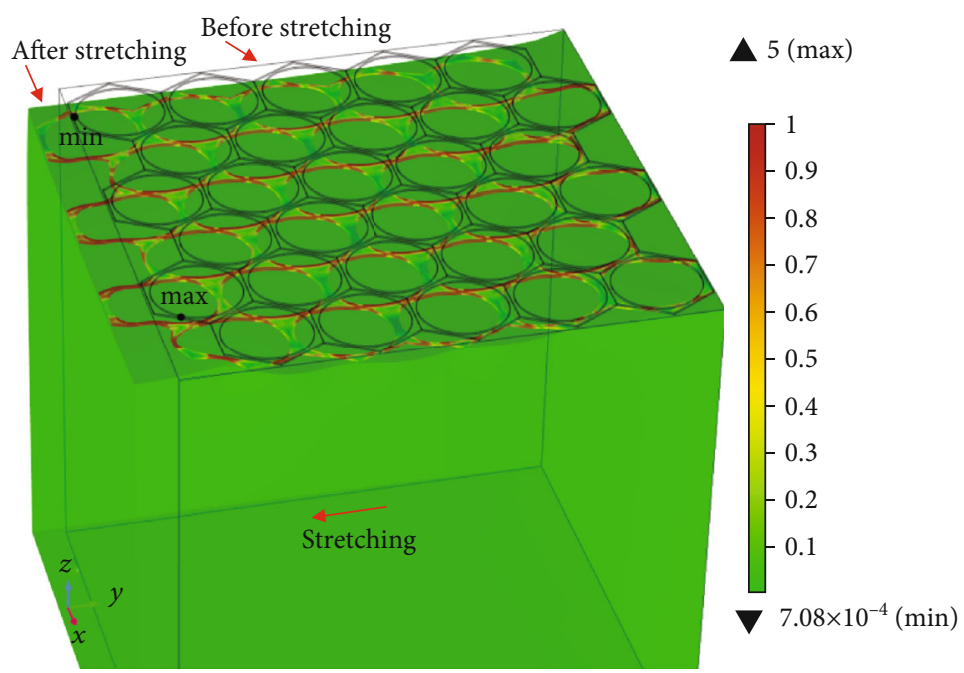

(b) Von Mises stress $(\mathrm{GPa})$ at displacement $=1.3831 \mathrm{E}-7 \mathrm{~m}$

FIGURE 3: Contour maps of Von Mises stress distribution of stretched (a) AuNMs and (b) AuNMs/PET with one side fixed and the other side under stretching in $-y$ direction. 


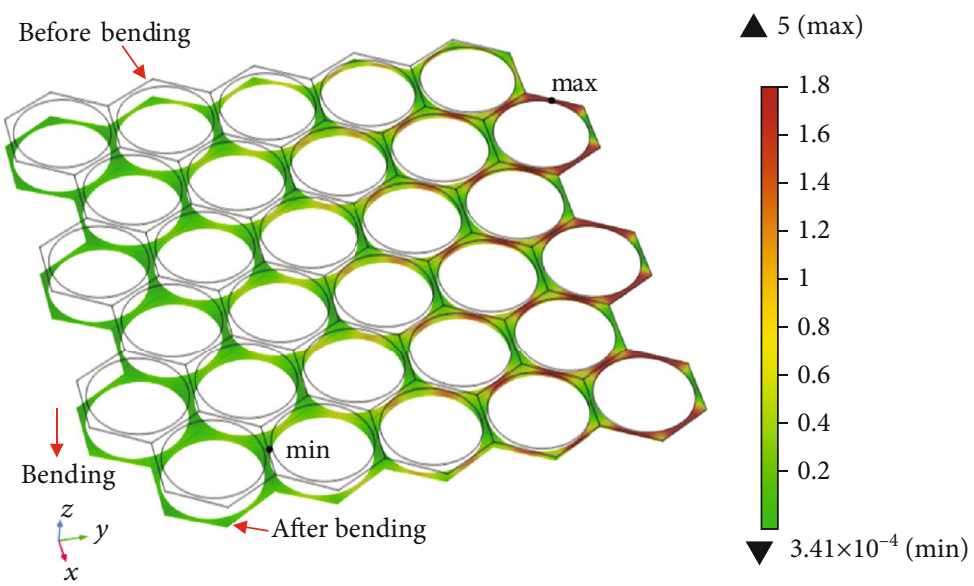

(a) Von Mises stress (GPa) at displacement $=2.6302 E-5 \mathrm{~m}$



(b) Von Mises stress (GPa) at displacement $=2.721 E-5 \mathrm{~m}$

Figure 4: Contour maps of Von Mises stress distribution of bent (a) AuNMs and (b) AuNMs/PET with one side fixed and the other side under bending in $-z$ direction.

Figure 5(a) shows the stress distribution and maximum and minimum stress points of the AuNMs/PET. This way of stretching causes two sides of PET to be subjected to the greatest force while the center point of PET is forced to zero as shown. Due to the fact that AuNMs with large Young's modulus cause very little deformation, while PET with small Young's modulus causes large deformation via releasing of the stress, the maximum stress value of AuNMs/PET appears in the side of AuNMs, and the minimum stress value appears in the middle of AuNMs, as shown in Figure 5(a). Figure 5(b) shows the displacement distribution and maximum and minimum displacement points of the AuNMs/PET. For the same reason, the maximum displacement of AuNMs/PET occurs at the side of the PET, and minimum displacement occurs at the center of PET, as shown in Figure 5(b). PET absorbs most of the externally induced physical strain on AuNMs.

The mechanical flexibility was examined here by studying the effect of strain on sheet resistance under mechanical deformation. To facilitate comparative analysis, a sample of AuNMs (thickness $\sim 20 \mathrm{~nm}$ ) on PET film (thickness $\sim 500 \mu \mathrm{m}$ ) with the same parameters as a model counterpart of numerical AuNMs/PET has been fabricated.

As shown as Figure 6(a), sheet resistance $R$ of AuNMs in the simulation under loading remains its original resistance $R_{0}$ until the tensile strain ratio reaches threshold point $1.2 \%$. The applied force under AuNMs/PET will lead to tensile strain, which can be accommodated by in-plane rotations and distortion of periodic nanomesh without inevitable structural fracture of AuNMs [22], followed by a dramatic increase of sheet resistance at strain beyond 1.2\%, which would eventually cause structural fracture of AuNMs and electroconductive failure. The experimental results with a threshold point of $1.8 \%$ show better flexible performance. This may be caused by the fact that the fabricated AuNMs with a size of several square centimeters can accommodate more tensile strain than the simulated model with a size of several square microns. Figure 6(b) shows the same trend, but better flexible performance, which is due to the fact that AuNMs can maintain the electroconductive state better 


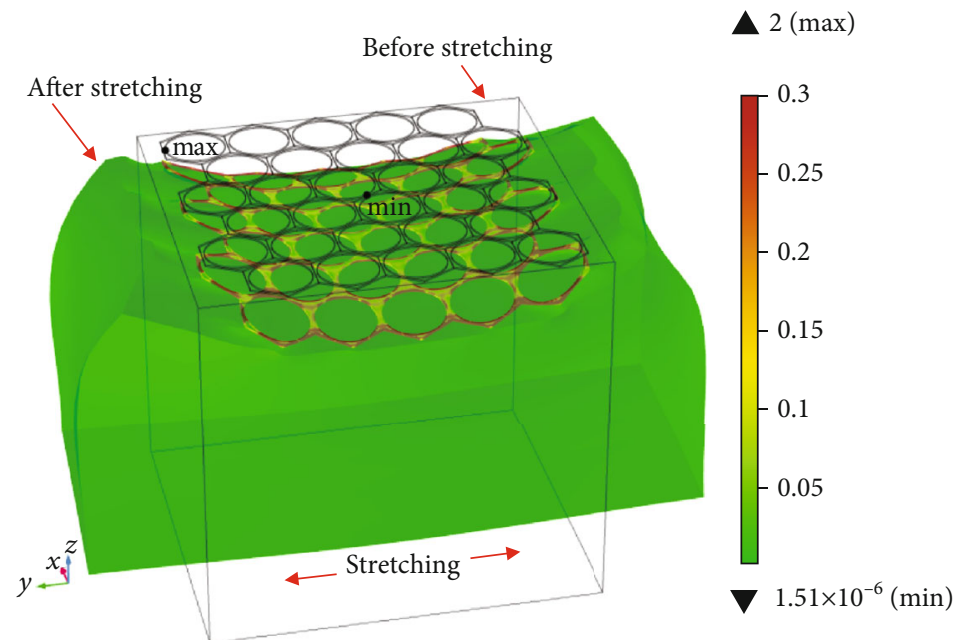

(a) Von Mises stress (GPa) and max/min: stress (GPa)



(b) Displacement $(\mu \mathrm{m})$ and $\max / \mathrm{min}$ : displacement $(\mu \mathrm{m})$

Figure 5: The flexibility performance of AuNMs/PET. (a) Contour maps of Von Mises stress distribution of AuNMs/PET. (b) Contour maps of displacement distribution of AuNMs on PET with both sides of PET under stretching in $y$ and $-y$ directions, respectively.

under compressive loading than tensile loading. It is not hard to find that the simulated results show good agreement with experimental results.

Finally, sheet resistance of the electrode under cyclic compressive and tensile bending tests was measured, which will assess the mechanical stability of the AuNMs. To make sure the bending test was carried out within a reasonable range, the bending radii ( 5 to $10 \mathrm{~mm}$ ) were chosen to apply enough strain for the bending test, depending on the electrode dimensions of width $=2 \mathrm{~cm}$ and length $=3 \mathrm{~cm}$. As shown in Figure 7, the electrode is bent to 400 cycles for 5 to $10 \mathrm{~mm}$ bending radii. When the pure Au films (thickness $\sim 20 \mathrm{~nm}$ ) are bent, there is no change in the resistance value until the number of bending of $\sim 40$ is reached (Figure 7). However, electrical failure suddenly occurred after the number of bending of $\sim 130$. The AuNMs can retain their initial sheet resistance until the number of bending reaches $\sim 350$. Thereafter, due to the plastic deformation of AuNMs, the sheet resistance of AuNMs increases gradually, which eventually leads to a complete breakdown of AuNMs. The
AuNMs show seven times higher tolerance than the pure $\mathrm{Au}$ films. The results indicate that AuNMs/PET shows good mechanical stability under both tensile loading and compressive loading.

\section{Conclusions}

In conclusion, we investigated the mechanical and structural response of AuNMs under mechanical deformation based on FEA simulation. The prepared AuNMs by the versatile NSL technique shows a uniform periodic nanostructure consistent with that of the AuNM model in the simulation. The simulated results of mechanical flexibility indicate that the PET substrate can release the stress of AuNMs under mechanical stretching and bending, the displacement of stretched AuNMs yields a $2 \%$ promotion, and the displacement of bent AuNMs yields a 3.5\% promotion under buffering of PET substrate at $5 \mathrm{GPa}$ yield strength of nanoscale $\mathrm{Au}$. The stress and displacement distribution of the AuNMs/PET was demonstrated. In the study of the effect of strain on sheet 


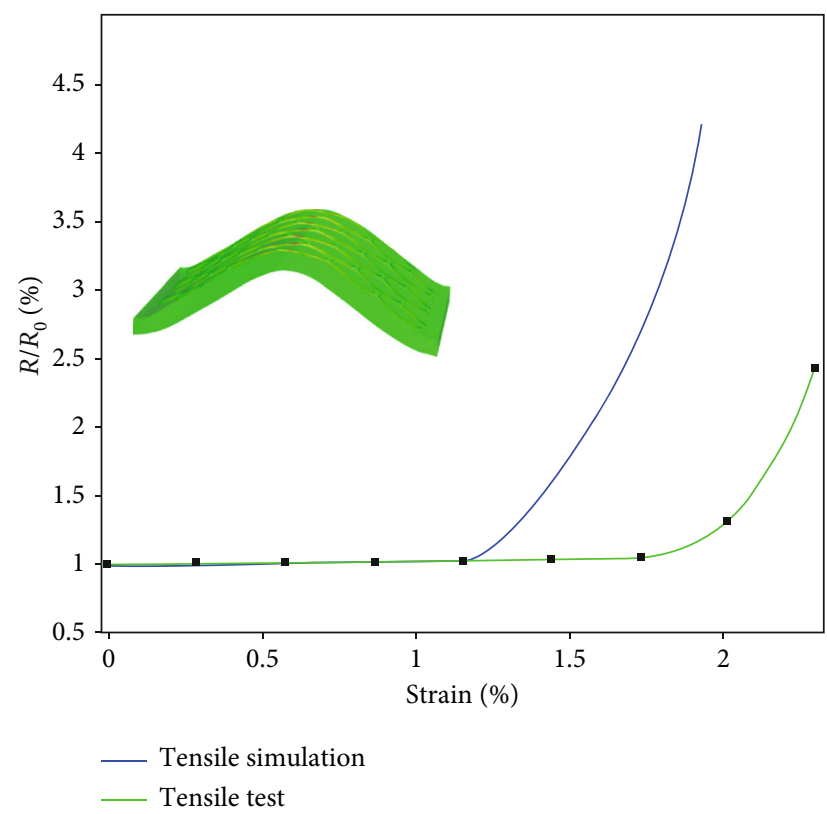

(a)

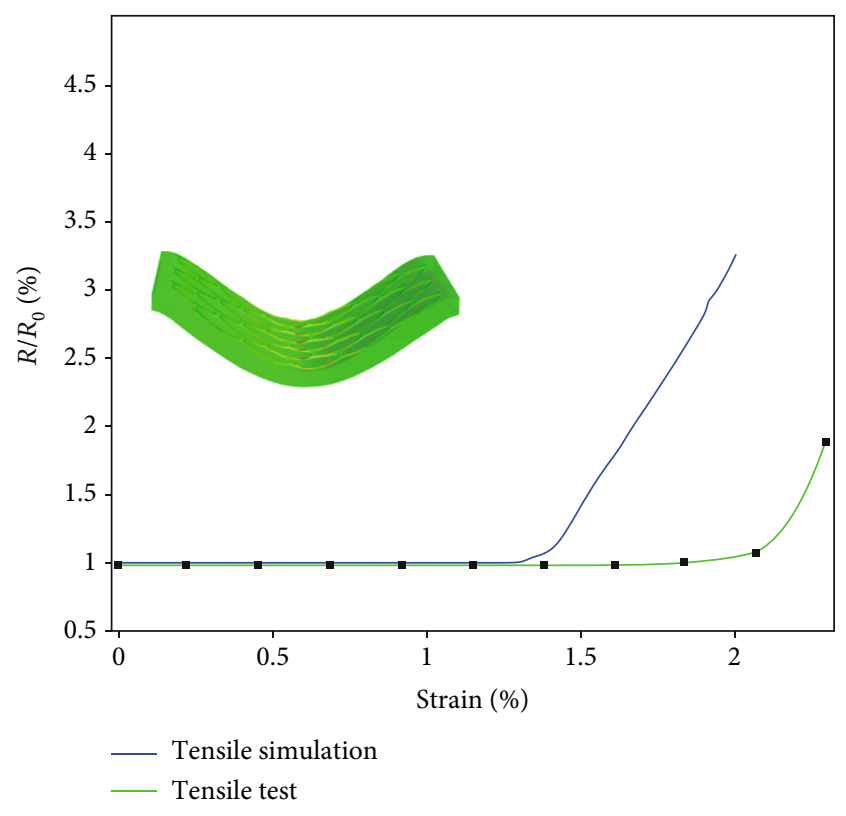

(b)

FIGURE 6: $R / R_{0}$ vs. the strain level for AuNMs/PET (where $R_{0}$ is the initial resistance under zero strain): (a) during tensile loading; (b) during compressive loading. The insets show maps of AuNMs/PET during compressive and tensile loading, respectively.

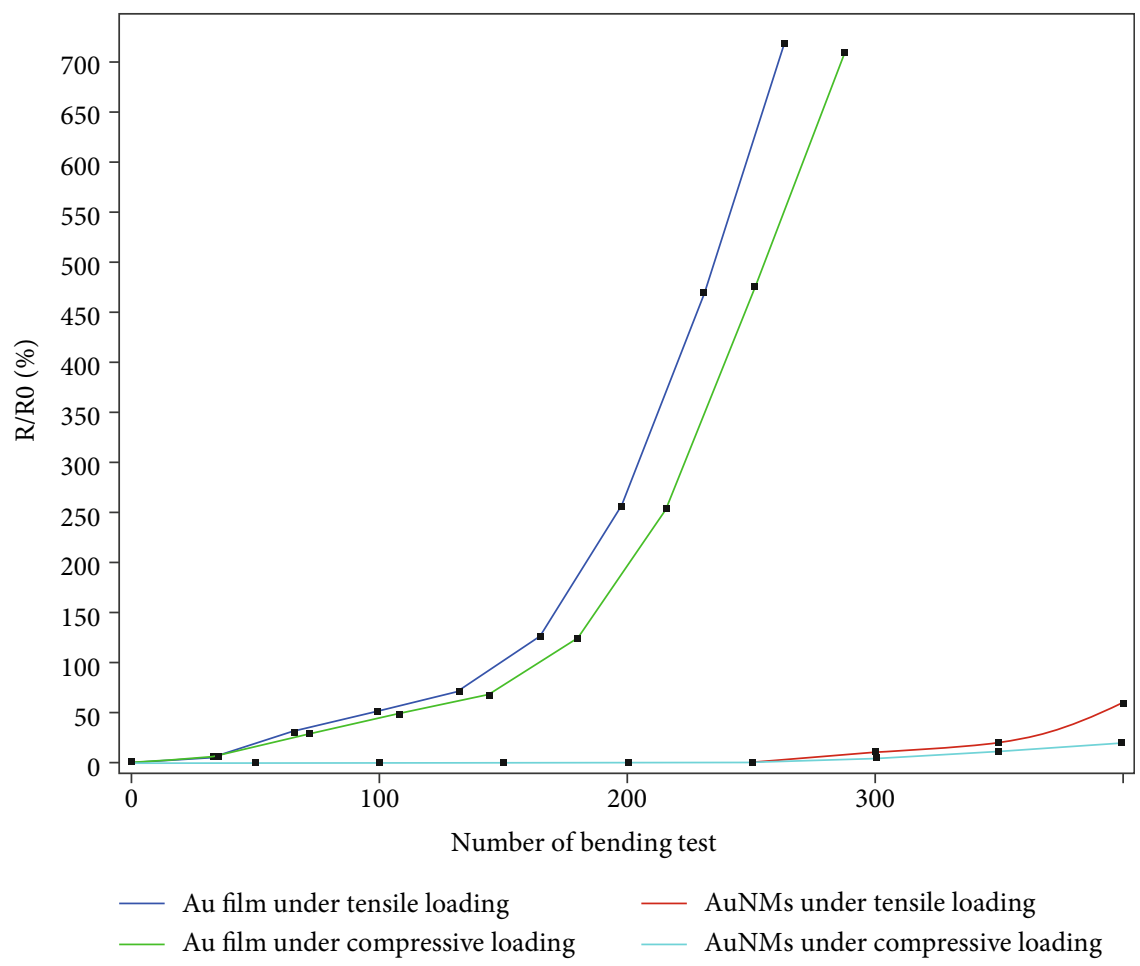

Figure 7: Sheet resistance vs. the number of bending of AuNMs during tensile loading and compressive loading, respectively. The electrode used has dimensions of width $=2 \mathrm{~cm}$, length $=3 \mathrm{~cm}$, and pristine sheet resistance $=33 \mathrm{ohm} / \mathrm{sq}$ (AuNMs) and $7 \mathrm{ohm} / \mathrm{sq}$ (Au films).

resistance under compressive and tensile loading, the simulated data shows good agreement with experimental results. Finally, cyclic compressive and tensile bending tests reveal the good mechanical stability of AuNMs. These results show the promising potential of using AuNMs in flexible electronics. 


\section{Data Availability}

The data used to support the findings of this study are available from the corresponding author upon request.

\section{Conflicts of Interest}

The authors declare no conflicts of interest.

\section{Authors' Contributions}

Li D.J. and Li G.M. contributed equally to this work. Li D.J., Li G.M., and Wan Q. designed the research; Xu W.X. fabricated the devices; Li D.J., Li G.M., Wan Q., and Liu J.H. performed data analysis; Li D.J., Li G.M., and Li Z.P. cowrote the manuscript while all other authors provided feedback.

\section{Acknowledgments}

The authors acknowledge the support from the Ph.D. research startup foundation of Hubei University of Science and Technology (BK202026, BK201819, and BK201801) and Scientific Research Project of Education Department of Hubei Province (B2019164 and B2019154).

\section{Supplementary Materials}

The file named "figure6a-simulation" is the simulated dataset in Figure 6(a). The file named "figure6a-test" is the experimental dataset in Figure 6(a). The file named "figure6bsimulation" is the simulated dataset in Figure 6(b). The file named "figure6b-test" is the experimentals dataset in Figure 6(b). The file named "figure7-compressive" is the experimental dataset under compressive loading in Figure 7. The file named "figure7-tensile" is the experimental dataset under tensile loading in Figure 7. (Supplementary Materials)

\section{References}

[1] D. R. Cairns, R. P. Witte II, D. K. Sparacin et al., "Straindependent electrical resistance of tin-doped indium oxide on polymer substrates," Applied Physics Letters, vol. 76, no. 11, pp. 1425-1427, 2000.

[2] J. H. M. Maurer, L. González-García, B. Reiser, I. Kanelidis, and T. Kraus, "Templated self-assembly of ultrathin gold nanowires by nanoimprinting for transparent flexible electronics," Nano Letters, vol. 16, no. 5, pp. 2921-2925, 2016.

[3] O. Inganäs, "Avoiding indium," Nature Photonics, vol. 5, no. 4, pp. 201-202, 2011.

[4] D. S. Hecht, L. Hu, and G. Irvin, "Emerging transparent electrodes based on thin films of carbon nanotubes, graphene, and metallic nanostructures," Advanced Materials, vol. 23, no. 13, pp. 1482-1513, 2011.

[5] T. Sun, C. Fei Guo, F. Cao et al., "A broadband solar absorber with $12 \mathrm{~nm}$ thick ultrathin a-Si layer by using random metallic nanomeshes," Applied Physics Letters, vol. 104, no. 25, p. 251119, 2014.

[6] R. Laskowski, P. Blaha, T. Gallauner, and K. Schwarz, "Singlelayer model of the hexagonal boron nitride nanomesh on the Rh(111) surface," Physical Review Letters, vol. 98, no. 10, 2007.
[7] H. Y. Jang, S.-K. Lee, S. H. Cho, J.-H. Ahn, and S. Park, "Fabrication of metallic nanomesh: Pt nano-mesh as a proof of concept for stretchable and transparent electrodes," Chemistry of Materials, vol. 25, no. 17, pp. 3535-3538, 2013.

[8] C. F. Guo, T. Sun, Q. Liu, Z. Suo, and Z. Ren, "Highly stretchable and transparent nanomesh electrodes made by grain boundary lithography," Nature Communications, vol. 5, no. 1, 2014.

[9] C. F. Guo, Y. Lan, T. Sun, and Z. Ren, "Deformation-induced cold-welding for self-healing of super-durable flexible transparent electrodes," Nano Energy, vol. 8, pp. 110-117, 2014.

[10] S. Huang, Y. Liu, Y. Zhao, Z. Ren, and C. F. Guo, "Flexible electronics: stretchable electrodes and their future," Advanced Functional Materials, vol. 29, no. 6, article 1805924, 2019.

[11] Z. H. Chen, R. Fang, W. Li, and J. Guan, "Stretchable transparent conductors: from micro/macromechanics to applications," Advanced Materials, vol. 31, no. 35, article 1900756, 2019.

[12] Y. Qiang, K. J. Seo, X. Zhao et al., "Bilayer nanomesh structures for transparent recording and stimulating microelectrodes," Advanced Functional Materials, vol. 27, no. 48, p. 1704117, 2017.

[13] B. Zhu, S. Gong, and W. Cheng, "Softening gold for elastronics," Chemical Society Reviews, vol. 48, no. 6, pp. 1668$1711,2019$.

[14] D. Zhao, S. Ke, Y. Hu, B. Wang, and P. Lu, "Optical bistability of graphene embedded in parity-time-symmetric photonic lattices," Journal of the Optical Society of America B: Optical Physics, vol. 36, no. 7, pp. 1731-1737, 2019.

[15] R. Vayron, V.-H. Nguyen, R. Bosc, S. Naili, and G. Haïat, "Assessment of the biomechanical stability of a dental implant with quantitative ultrasound: a three-dimensional finite element study," The Journal of the Acoustical Society of America, vol. 139, no. 2, pp. 773-780, 2016.

[16] D. Zhao, Z. Q. Wang, H. Long, K. Wang, B. Wang, and P. X. $\mathrm{Lu}$, "Optical bistability in defective photonic multilayers doped by graphene," Optical and Quantum Electronics, vol. 49, no. 4, 2017.

[17] R. Vayron, V. H. Nguyen, R. Bosc, S. Naili, and G. Haïat, "Finite element simulation of ultrasonic wave propagation in a dental implant for biomechanical stability assessment," Biomechanics and Modeling in Mechanobiology, vol. 14, no. 5, pp. 1021-1032, 2015.

[18] J. F. Zhu, B. Q. Zeng, and Z. Wu, "Enhanced broadband optical transmission through ultrathin metallic nanomesh," Journal of Electromagnetic Waves and Applications, vol. 26, no. 2-3, pp. 342-352, 2012.

[19] Z. Li, G. Wang, Z. Li, Z. Cheng, G. Zhou, and S. Li, "Flexible transparent electrodes based on gold nanomeshes," Nanoscale Research Letters, vol. 14, no. 1, p. 132, 2019.

[20] J. Yan, L. Yang, M.-F. Lin, J. Ma, X. Lu, and P. S. Lee, “Polydopamine spheres as active templates for convenient synthesis of various nanostructures," Small, vol. 9, no. 4, pp. 596-603, 2013.

[21] A. Plettl, F. Enderle, M. Saitner et al., "Non-close-packed crystals from self-assembled polystyrene spheres by isotropic plasma etching: adding flexibility to colloid lithography," Advanced Functional Materials, vol. 19, no. 20, pp. 32793284, 2009.

[22] A. Todoroki, K. Yamada, Y. Mizutani, Y. Suzuki, and R. Matsuzaki, "Impact damage detection of a carbon-fibrereinforced-polymer plate employing self-sensing timedomain reflectometry," Composite Structures, vol. 130, pp. 174-179, 2015. 